\title{
Evaluation and prevention of formation damage in offshore sandstone reservoirs in China
}

\author{
Yang Shenglai ${ }^{1}{ }^{*}$, Sheng Zhichao ${ }^{1}$, Liu Wenhui², Song Zhixue ${ }^{2}$, Wu Ming ${ }^{1}$ \\ and Zhang Jianwei ${ }^{1}$
}

${ }^{1}$ Key Laboratory of Petroleum Engineering, Ministry of Education, China University of Petroleum, Beijing 102249, China

${ }^{2}$ Bohai Oil Research Institute, CNOOC, Tianjin 230045, China

\begin{abstract}
Reduction in water injectivity would be harmful to the waterflood development of offshore sandstone oil reservoirs. In this paper the magnitude of formation damage during water injection was evaluated by analyzing the performance of water injection in the Bohai offshore oilfield, China. Two parameters, permeability reduction and rate of wellhead pressure rise, were proposed to evaluate the formation damage around injection wells. The pressure performance curve could be divided into three stages with different characteristics. Analysis of field data shows that formation damage caused by water injection was severe in some wells in the Bohai offshore oilfield, China. In the laboratory, the content of clay minerals in reservoir rock was analyzed and sensitivity tests (including sensitivity to water, flow rate, alkali, salt and acid) were also conducted. Experimental results show that the reservoir had a strong to medium sensitivity to water (i.e. clay swelling) and a strong to medium sensitivity to flow rate, which may cause formation damage. For formation damage prevention, three injection schemes of clay stabilizer (CS) were studied, i.e. continuous injection of low concentration CS (CI), slug injection of high concentration CS (SI), and slug injection of high concentration CS followed by continuous injection of low concentration CS (SI-CI). Core flooding experiments show that SI-CI is an effective scheme to prevent formation damage and is recommended for the sandstone oil reservoirs in the Bohai offshore oilfield during water injection.
\end{abstract}

Key words: Sandstone reservoir, formation damage, water injection, clay stabilizer

\section{Introduction}

The water injection rate was designed at $300-500 \mathrm{~m}^{3} / \mathrm{d}$ per well in the Bohai offshore oilfield, China. A problem encountered while water was injected at a constant rate was that the wellhead pressure of the injection well increased gradually with time. In some wells, bottomhole pressure reached fracture pressure within one year.

Previous research on onshore oilfields indicates that formation damage is the main reason for wellhead pressure rise, and clay stabilizer can be used to prevent or alleviate formation damage.

Water injectivity decline is common in waterflooding fields. Sharma and Pang (1997) presented a case study for an offshore Gulf of Mexico water injection project. Five typical offshore wells with rapid decline in injectivity were observed. In the injection wells that were not fractured, a decline in injectivity was experienced even for the injection of relatively clean water. Fracturing the injection wells appeared to be the only feasible way of substantially increasing the life of such injectors.

\footnotetext{
*Corresponding author. email: yangsl@cup.edu.cn
} Received March 20, 2008
The permeability damage mechanisms can be grouped into three general categories: mechanical fines migration, brine sensitivity and geochemical transformations. Molenaar and Huyghe (1996) studied the particle movement and deposition in porous media. Chang (1997) and Civan (1999, 2001) discussed the mechanism of swelling and drew a conclusion that the charge defect was the foremost reason for clay swelling. Leone and Scott (1988) conducted core flooding experiments with preserved core samples to evaluate the influences of brine composition, temperature and flow rate on permeability damage in cores. The significance of damage during brine injection was observed to be a strong function of mineralogy and injection rate. Permeability loss was $>60 \%$ in cores from some sand intervals. The occurrence of a critical velocity, along with other observations, indicated that the primary damage mechanism was fines migration.

Moghadasi et al (2004) investigated the possible reason for injectivity loss in a typical Iranian oilfield. It was observed that the build-up of flow resistance was mostly due to frontal face plugging. Al-Hamadah (1995) and Bedrikovetsky et al (2005) studied the reason for injectivity decline during seawater injection. The temperature of the seawater injected is significantly lower than the reservoir temperature and also lower than the previously injected water obtained from aquifers. Therefore, a decline in injectivity is expected as a 
result of higher oil viscosity due to the lower temperature of injection water. Actual data collected from water injection wells showed a significant decline in injectivity after conversion to seawater.

To prevent formation damage, chemical injection is the common method used (Xiong and Holditch, 1995). Norman and Smith (2000) studied clay stabilization with $\mathrm{KOH}$. The KOH-clay chemical reaction permanently alters the clay chemistry so that the clay minerals are unaffected by changes in water composition. Several field case histories demonstrated that $\mathrm{KOH}$ can mitigate the effects of both clay swelling and fines migration and a single $\mathrm{KOH}$ treatment is effective over the economic life of the well. Nasr-El-Din et al (1998) conducted an experimental study to design an effective procedure of treatment with clay stabilizer. The effects of stabilizer concentration, soaking time, and acids on core permeability were investigated in detail.

In this paper, mineral sensitivities of formation core to water, flow rate and salt were measured in the laboratory. The magnitude of formation damage was evaluated by field data. For formation protection, test on three injection schemes for clay stabilizer were conducted and the effects of damage prevention was compared.

\section{Evaluation of formation damage}

\subsection{Performance of water injection}

The history curves of injection rate and wellhead pressure for a typical well, Well No. 1, in the Bohai offshore oilfield, China are shown in Fig. 1. This shows that the injection pressure increased gradually with time when water was injected at a constant rate. It was the water injected that resulted in an increase in pressure. So the cumulative volume of water injected was responsible for pressure increase. Fig. 2 shows that the wellhead pressure rise and injectivity decline was due to the increase in cumulative volume of water injected into the well. Fig. 3 shows that the relative injectivity changed with increasing cumulative volume of water injected. For example, after $170 \times 10^{3} \mathrm{~m}^{3}$ of water were injected into the well, the injectivity was only $40 \%$ of the initial value.

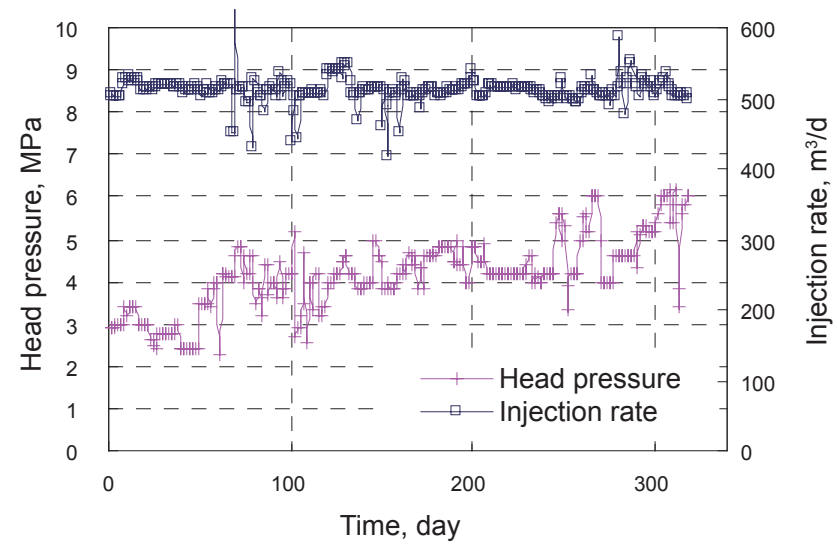

Fig. 1 Performance of water injection for Well No.1

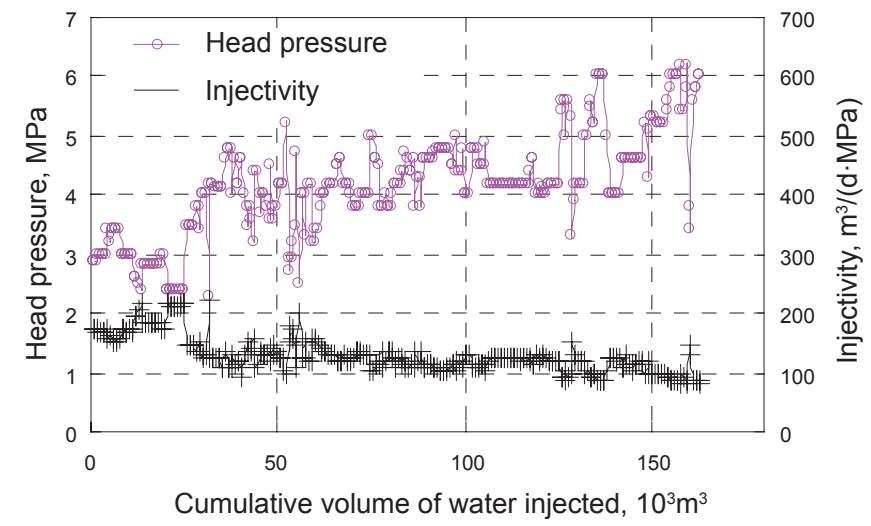

Fig. 2 Injectivity decline curve for Well No.1

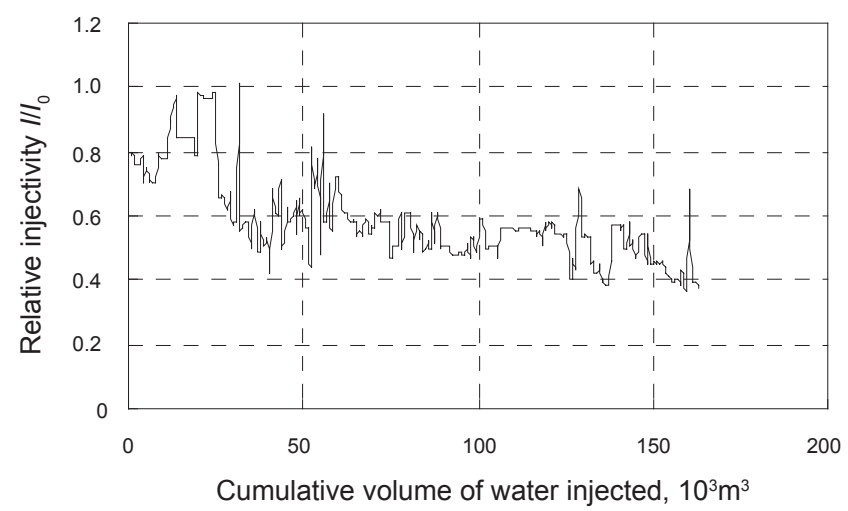

Fig. 3 Relative injectivity for Well No. 1

Figs. 4-6 show the field data for Well No. 2. Similarly, the injectivity declined to $40 \%$ of the original value after 170 $\times 10^{3} \mathrm{~m}^{3}$ of water were injected into the Well No. 2, as shown in Fig. 6.

The rise of wellhead pressure may be caused by the volume change of fluid flow in reservoir or by formation damage. Oilfield data indicated that the daily output was larger than the daily injection in the Bohai offshore oilfield, and the cumulative output was higher than cumulative input, so reservoir voidage was occurring. Under this condition, the rise of injection pressure indicated that formation damage, which may be caused by suspended particles blocking, clay swelling, and clay particles migration during water injection, had happened in the vicinity of injection wells. The reservoir clay usually contains montmorillonite, a mixture of illite and montmorillonite and other minerals. If the water injected is not compatible with the minerals in the payzone, montmorillonite tends to swell, and kaolinite particles will disperse and migrate. This will eventually reduce the permeability and injectivity of injection wells. If the water injection rate is kept constant, the wellhead pressure will accordingly increase because of permeability reduction. The curves in Figs. 1 and 4 show the change of wellhead pressure with time, and also indicate the extent of formation damage with time. The cumulative volume of water injected increased with time, so the magnitude of formation damage increased consequently (Figs. 2 and 5). 


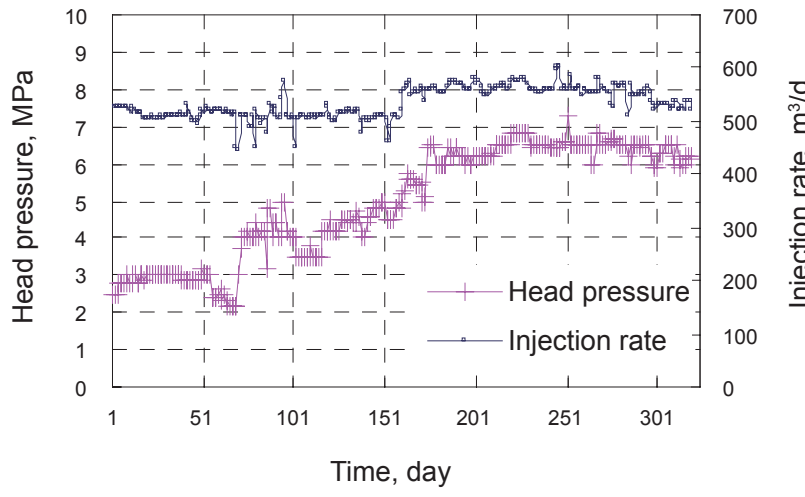

Fig. 4 Performance of water injection for Well No. 2

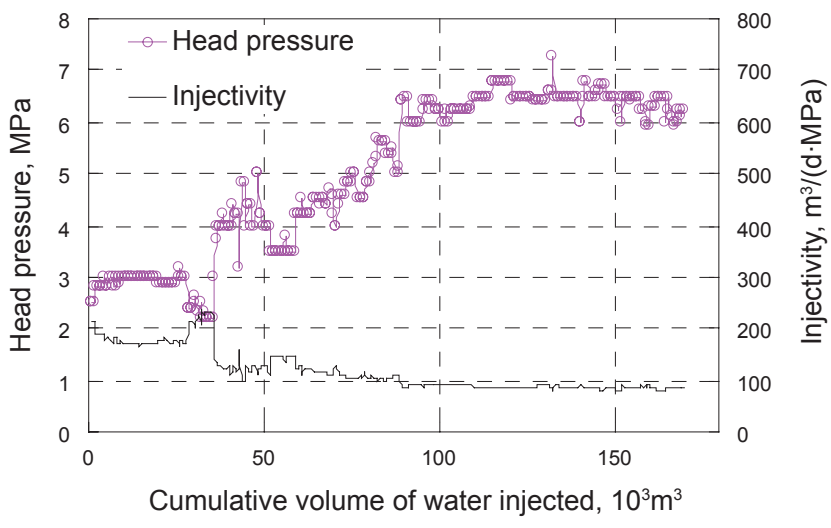

Fig. 5 Injectivity decline curve for Well No. 2

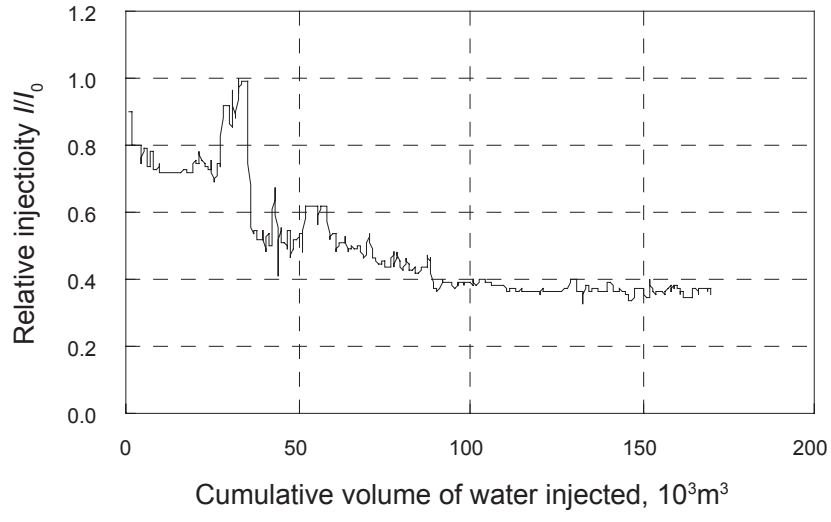

Fig. 6 Relative injectivity for Well No. 2

\subsection{Evaluation of formation sensitivity}

To understand the mechanism of formation damage, the content of sensitive minerals in sandstone, pore structure and fluid properties were analyzed. The mineral content is listed in Table 1.

Tests on sensitivity to water, salt, flow rate and alkali were also conducted by using core samples from the Bohai offshore oilfield, according to Chinese National Standard SY/ T5358-2002 'Evaluation Method of the Formation Sensitivity by Core Flow Experiment'. Experimental results (Table 2) indicate that the formation has a strong to medium sensitivity to water and a strong to medium sensitivity to flow rate. The combined action of water/flow rate sensitivities is the main reason for formation damage and water injectivity decline.

Table 1 Mineral content

\begin{tabular}{cccccc}
\hline \multirow{2}{*}{ Layer } & Total percentage of clay & \multicolumn{4}{c}{ Content, \% } \\
\cline { 3 - 5 } & 23.5 & Montmorillonite & Kaolinite & Illite & Chlorite \\
\hline Layer 1 & 24.7 & 4.72 & 11.15 & 70.09 & 14.05 \\
Layer 2 & 24.1 & 35.23 & 25 & 39.77 & 0 \\
\hline Average & 19.98 & 18.08 & 54.93 & 7.03 \\
\hline
\end{tabular}

Table 2 Formation sensitivities

\begin{tabular}{ccc}
\hline Experiment & Sensitivity & Evaluation \\
\hline 1 & Water & Strong-medium \\
2 & Flow rate & Strong-medium \\
3 & Salt & Medium \\
4 & Alkali & Weak \\
5 & Acid & Weak \\
\hline
\end{tabular}

\subsection{Evaluation of pressure rise}

Based on oilfield data and theoretical study, the pressure rise with cumulative volume of water injected could be divided into 3 stages according to the rate of pressure rise (as shown in Figs. 7 and 8):

1) Low-pressure stage: In this stage, the injected water filled up the pressure drop funnel formed during oil production. So the injection pressure was at a low level.

2) Damage stage: In the vicinity of the wellbore, sensitive minerals began to react with water injected and formation permeability near the well declined. Formation near the wellbore was damaged at this stage and pressure increased sharply.

3) High-pressure stage: As more water was injected into 
the formation, the water-swept area enlarged. Damage effects spread to a larger area away from the well. Injection pressure was at a high level.

Taking Well No. 2 as an example, initial water injection pressure was near $3 \mathrm{MPa}$. About 3 months later, formation damage came to effect and the pressure started to rise. After about $1.0 \times 10^{5} \mathrm{~m}^{3}$ of water had been injected into the well (Stage 3), the pressure was near $6 \mathrm{MPa}$.

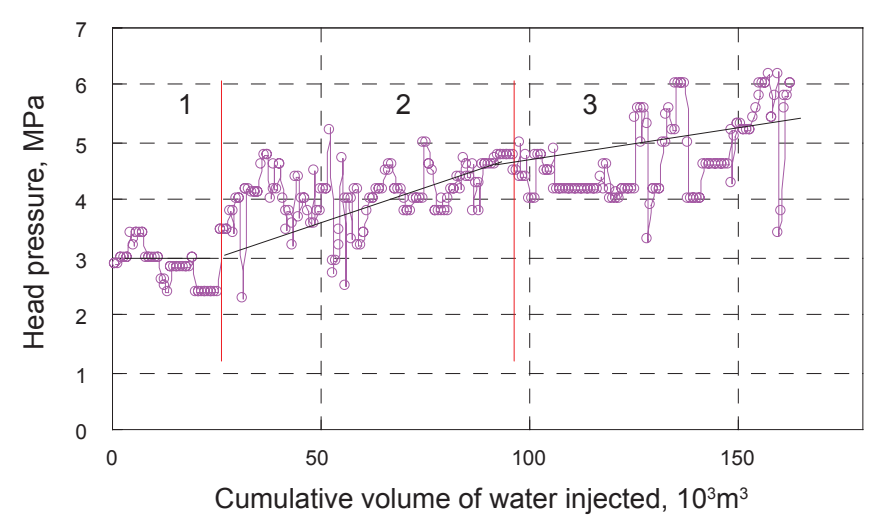

Fig. 7 Stages of injection pressure rise for Well No. 1

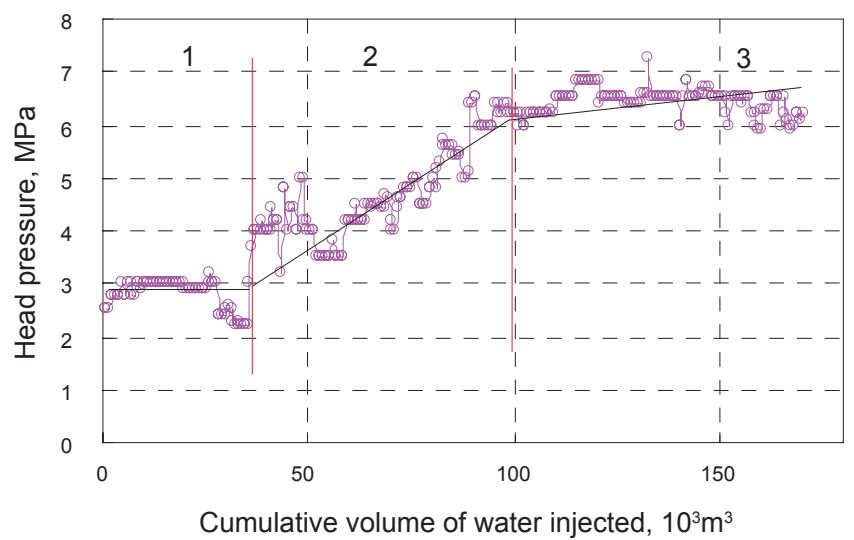

Fig. 8 Stages of injection pressure rise for Well No. 2

Formation damage is proportional to the cumulative volume of water injected. The injection pressure has a statistical dependence on the cumulative volume of water injected. To describe the rate of pressure rise and the rate of formation damage over a specified period of time, the rate of wellhead pressure (or injection pressure) rise is defined as follows:

$$
\alpha=\frac{\Delta p_{\mathrm{wH}}}{\Delta V_{\mathrm{I}}}
$$

where $\quad \alpha$ is the rate of injection (wellhead) pressure rise, $\mathrm{MPa} / 10^{3} \mathrm{~m}^{3} ; \Delta V_{\mathrm{I}}$ is the cumulative volume of water injected, $10^{3} \mathrm{~m}^{3} ; \Delta p_{\mathrm{WH}}$ is pressure difference of water injection, $\mathrm{MPa}$

Parameter $\alpha$ is applicable to reservoirs with injection and production voidage nearly balancing, which can be calculated from the curve of cumulative volume of water injected versus wellhead pressure (Figs. 7 and 8). To linearly fit this curve over a period of time, the slope is the average rate of injection pressure rise in this period. The physical significance of $\alpha$ is the value of wellhead pressure increment per cubic meter water injected. The values of $\alpha$ for 3 stages were calculated separately and the results are listed in Table 3.

Table 3 Rate of wellhead pressure rise

\begin{tabular}{cccc} 
& & & $\left(\mathrm{MPa} / 10^{3} \mathrm{~m}^{3}\right)$ \\
\hline Stage & Well No.1 & Well No.2 & Well No.3 \\
\hline 1 & 0.0012 & 0.0056 & 0.0040 \\
2 & 0.0216 & 0.0465 & 0.0457 \\
3 & 0.0123 & 0.0100 & 0.0099 \\
\hline Average & 0.0137 & 0.0227 & 0.0142 \\
\hline
\end{tabular}

Fig. 9 illustrates the average rate of wellhead pressure rise, which was calculated from the data acquired in the whole injection process.

In the oilfield, the rate of wellhead pressure rise can also be used in treatment decision-making. If the rate of wellhead pressure rise is greater than $0.0142 \mathrm{MPa} / 10^{3} \mathrm{~m}^{3}$ within a month, treatment with clay stabilizer during water injection is necessary.

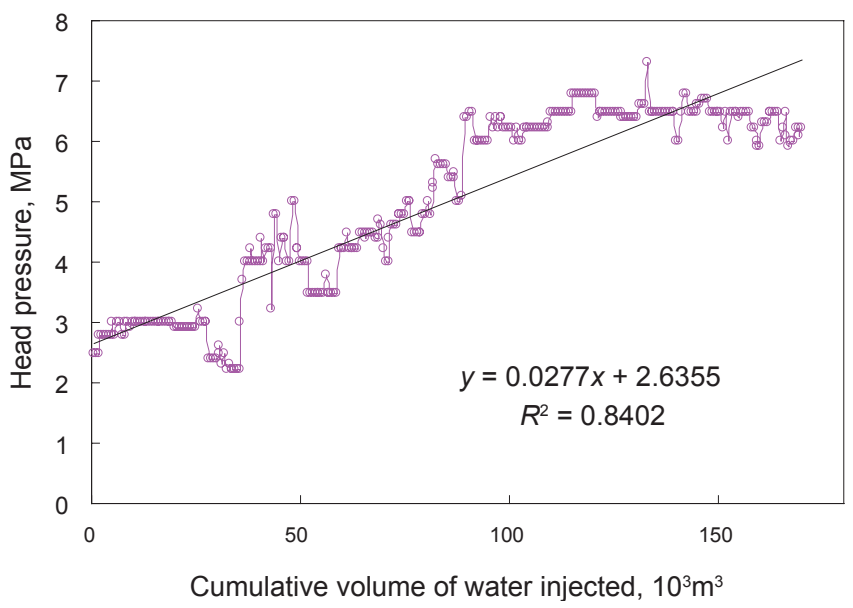

Fig. 9 Average rate of wellhead pressure rise, Well No. 2

\subsection{Evaluation of formation damage}

Formation damage is defined as the reduction of the flow capacity of the reservoir formation:

$$
K^{*}=\frac{I_{0}-I_{1}}{I_{0}}
$$

where $I_{0}$ is the initial injectivity, $\mathrm{m}^{3} /(\mathrm{d} \cdot \mathrm{MPa}) ; I_{1}$ is the present injectivity, $\mathrm{m}^{3} /(\mathrm{d} \cdot \mathrm{MPa})$.

Because the formation damage occurs in the vicinity 
of the well bore, the injectivity before and after formation damage can be expressed with Darcy's equation for plane radial flow:

$$
I_{1}=\frac{Q_{0}}{\Delta P_{0}}=\frac{2 \pi K_{0} h}{\mu \ln \left(r_{\mathrm{e}} / r_{\mathrm{w}}\right)}
$$

where $Q_{0}$ is initial injection flow rate, $\mathrm{m}^{3} / \mathrm{d} ; \Delta P_{0}$ is the initial pressure difference of water injection, $\mathrm{MPa} ; K_{0}$ is the primary permeability before damage, $\mu \mathrm{m}^{2} ; h$ is the effective thickness of pay zone, $\mathrm{m} ; \mu$ is the water viscosity, $\mathrm{mPa} \cdot \mathrm{s} ; r_{\mathrm{e}}$ is the flow boundary radius, $\mathrm{m} ; r_{\mathrm{w}}$ is the well radius, $\mathrm{m}$.

$$
I_{1}=\frac{Q_{1}}{\Delta P_{1}}=\frac{2 \pi K_{1} h}{\mu \ln \left(r_{\mathrm{e}} / r_{\mathrm{w}}\right)}
$$

where $Q_{1}$ is the present injection flow rate, $\mathrm{m}^{3} / \mathrm{d} ; \Delta P_{1}$ is the present pressure difference of water injection, $\mathrm{MPa} ; K_{1}$ is the permeability after damage, $\mu \mathrm{m}^{2}$.

If $r_{\mathrm{e}}, h, r_{\mathrm{w}}$ and $\mu$ are unchanged, substituting Eqs. (3) and (4) into Eq. (2) gives:

$$
K^{*}=\frac{K_{0}-K_{1}}{K_{0}}
$$

Therefore, permeability reduction can be used to evaluate the formation damage.

If $Q$ is unchanged (i.e. $Q_{1}=Q_{0}$ ), Eq. (2) can be rewritten as follows:

$$
K^{*}=\frac{\Delta P_{1}-\Delta P_{0}}{\Delta P_{1}}
$$

According to fluid mechanics, $\Delta p$ is approximately equal to the wellhead pressure $P$. So Eq. (6) can be simplified as follows:

$$
K^{*}=\frac{P_{1}-P_{0}}{P_{1}}
$$

where $P_{0}$ is the initial wellhead pressure, $\mathrm{MPa} ; P_{1}$ is the present wellhead pressure, MPa.

With Eq. (7), the magnitude of formation damage was evaluated from the values of injection pressures. Table 4 shows that the permeability reduction was $0.489-0.692$, indicating severe formation damage.

Table 4 Evaluation of formation damage

\begin{tabular}{cccc}
\hline \multirow{2}{*}{ Well } & \multicolumn{2}{l}{ Head pressure, MPa } & Permeability reduction \\
\cline { 2 - 3 } & Low & High & \\
\hline 1 & 4.5 & 8.8 & 0.489 \\
2 & 1.6 & 5.2 & 0.692 \\
3 & 2.2 & 5 & 0.560 \\
\hline
\end{tabular}

\section{Formation protection technology - Injection scheme of clay stabilizer}

The main method of protecting reservoir formations is to inject clay stabilizer (CS) during water injection. The organiccation-polymer type of clay stabilizer is commonly used in China. Both technical and economic factors should be taken into account to design an effective clay stabilizer treatment. In this paper, core flood experiments (reservoir formation cores used) were conducted by using different injection schemes, i.e., continuous injection of low-concentration of CS (CI), slug injection of high-concentration of CS (SI) and slug injection of high-concentration of CS followed by continuous injection of low-concentration of CS (SI-CI).

\subsection{Experimental apparatus and materials}

The core flooding system CFS-100 made by Core Lab Co. was used to evaluate formation damage. The flow diagram of apparatus is shown in Fig. 10.

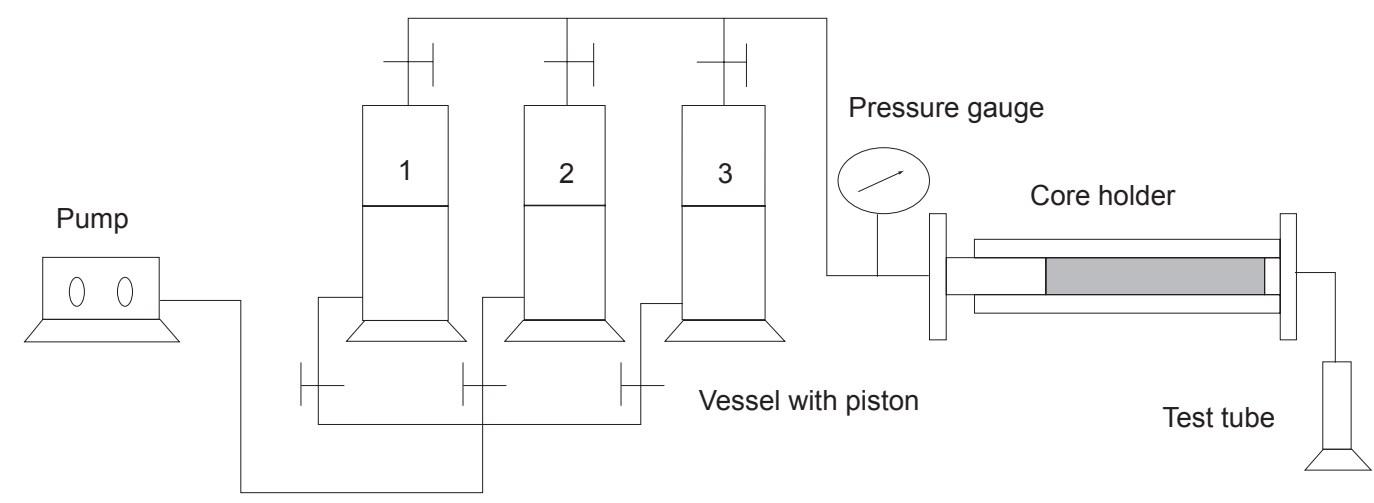

Fig. 10 Flow diagram of core flooding experiment 
Natural core samples from the Bohai offshore oilfield were used. The plug cores, $25 \mathrm{~mm}$ in diameter and 50-70 $\mathrm{mm}$ in length, were cleaned with solvent, then placed in a thermostat dry box at $85 \pm 1{ }^{\circ} \mathrm{C}$ for 6 hours to remove the solvent. After cooling to room temperature in a dry container, the plug cores can be used and their properties are listed in Table 5.

Table 5 Properties of plug cores used

\begin{tabular}{ccccc}
\hline Core & $\begin{array}{c}\text { Diameter } \\
\mathrm{mm}\end{array}$ & $\begin{array}{c}\text { Length } \\
\mathrm{mm}\end{array}$ & $\begin{array}{c}\text { Porosity } \\
\%\end{array}$ & $\begin{array}{c}\text { Permeability } \\
10^{-3} \mu \mathrm{m}^{2}\end{array}$ \\
\hline 1 & 25 & 75.83 & 25.11 & 45.2 \\
2 & 25 & 70.51 & 31.31 & 50.3 \\
3 & 25 & 63.61 & 35.95 & 63.8 \\
4 & 25 & 77.60 & 21.26 & 15.4 \\
\hline
\end{tabular}

Formation water, field injection water and clay stabilizer solutions (stabilizer concentration of $2 \%$ and $0.2 \%$ ) were prepared and the properties of these liquids used are shown in Table 6.

Table 6 Properties of liquids injected

\begin{tabular}{ccc}
\hline Liquids & $\begin{array}{c}\text { Density } \\
\mathrm{g} / \mathrm{cm}^{3}\end{array}$ & $\begin{array}{c}\text { Viscosity } \\
\text { MPa } \cdot \mathrm{s}\end{array}$ \\
\hline $0.2 \%$ clay stabilizer solution & 1.0045 & 1.207 \\
$2.0 \%$ clay stabilizer solution & 1.0260 & 1.216 \\
Formation water & 1.0067 & 1.085 \\
Field injection water & 1.0233 & 1.114 \\
\hline
\end{tabular}

\subsection{Experimental procedures}

Four tests were designed and conducted at room temperature in order to compare the effects of different clay stabilizer inject schemes. The liquids were injected at a flow rate of $0.6 \mathrm{~cm}^{3} / \mathrm{min}$ and the same volume of clay stabilizer was injected into the core in each test.

Test 1 (Conventional water injection, without any clay stabilizer): Formation water was injected to measure the permeability of plug core. Then 30 pore volume (PV) of field injection water was injected. The formation damage caused by field injection water was evaluated, and this can be used as a reference of original formation damage.

Test 2 (Slug injection of $2 \%$ stabilizer solution, SI): Formation water was first injected to measure the permeability of plug core. Then one pore volume of $2 \%$ clay stabilizer solution was injected into the core, followed by 10 PV of field injection water. These two liquids were alternately injected for 3 cycles.

Test 3 (Continuous injection of $0.2 \%$ stabilizer solution, CI): Formation water was injected to measure the permeability of core. Then $30 \mathrm{PV}$ of $0.2 \%$ clay stabilizer solution was continuously injected into the core.

Test 4 (Slug injection of 2\% stabilizer solution followed by continuous injection of $0.2 \%$ stabilizer solution, SI-CI): Formation water was injected to measure the permeability of core. Then $1 \mathrm{PV}$ of $2 \%$ clay stabilizer solution was injected into the core, followed by $20 \mathrm{PV}$ of $0.2 \%$ clay stabilizer solution and $9 \mathrm{PV}$ of formation water was injected in the end.

During experiments, the data including time, flow rate and pressure were recorded and the values of permeability and permeability reduction were calculated.

\subsection{Experimental results and discussion}

In case of the SI scheme, the permeability reduction $K^{*}$ was relatively low and increased slowly during the first injection of clay stabilizer solution, but a significant reduction of permeability was observed in the following process of water injection. Compared with the first cycle of injection, during the 2nd and 3rd cycles of injection, the permeability reduction $K^{*}$ increased more slowly (Fig. 11).

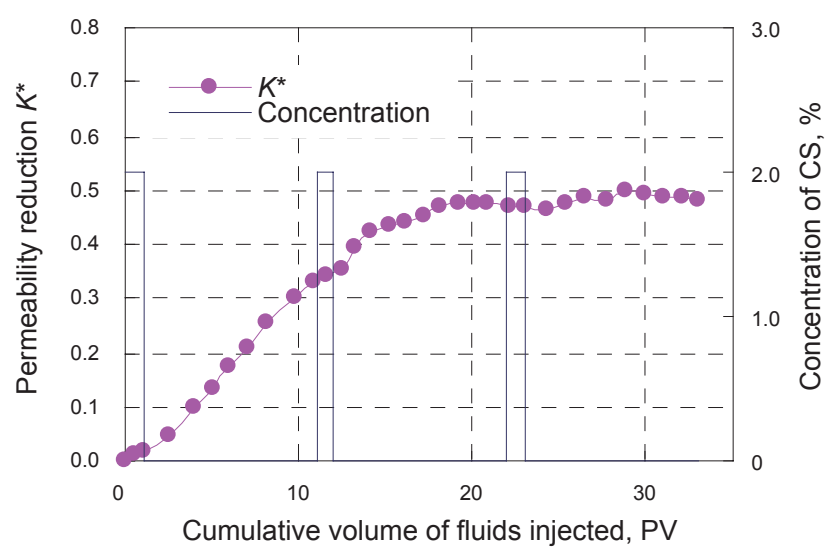

Fig. 11 Injection curves for SI scheme

Fig. 12 presents the permeability reduction versus cumulative fluid injected during CI scheme. At the beginning, permeability reduction increased sharply, however, after 4.5 PV of $0.2 \%$ stabilizer solution was injected into the core, permeability reduction changed slowly with the continuous injection of $0.2 \%$ stabilizer solution.

Fig. 13 presents the permeability reduction versus cumulative fluid injected during SI-CI process. Point A shows that the $2 \%$ clay stabilizer absorbed on the pore surface could alleviate or delay formation damage. This indicates that in order to allow the clay stabilizer to be more effectively adsorbed on the pore surface, CS solution should be injected at a low flow rate or the well should be shut-off for a period of time. 


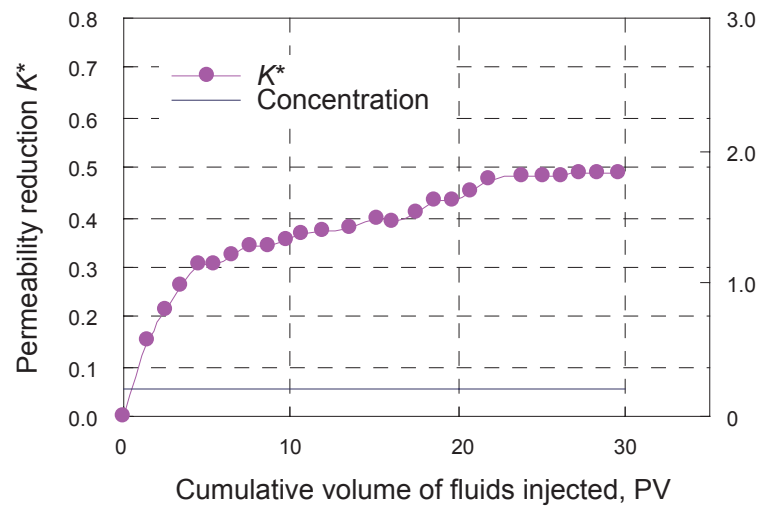

Fig. 12 Injection curves for CI scheme

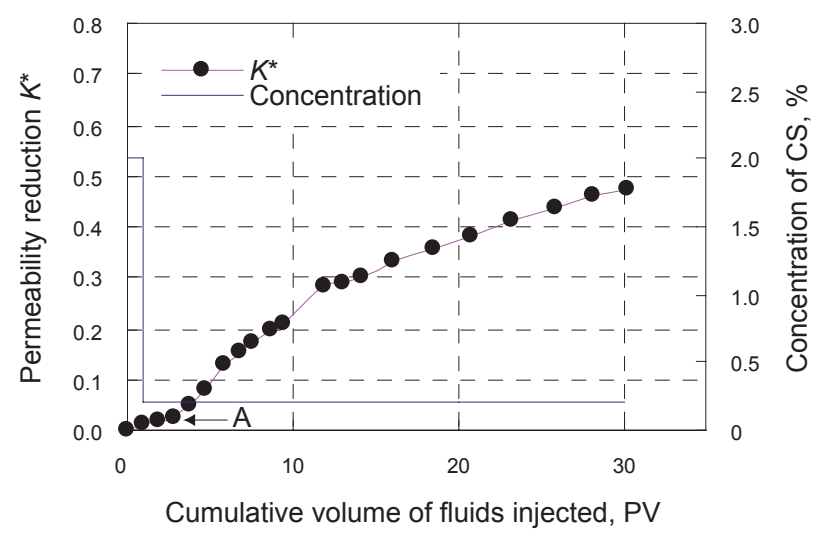

Fig. 13 Injection curves for SI-CI scheme

Comparison of different injection schemes (in Fig. 14) shows that the addition of clay stabilizer into injection water could effectively reduce formation damage. For the CI scheme, permeability reduction was relatively higher than other schemes at the initial stage. For the SI scheme, at the early stage the permeability reduction was lower than that in the CI scheme, but at the middle stage, the permeability reduction was higher than that in the CI scheme.

For the SI-CI scheme, permeability reduction increased slowly and finally kept at a relatively low level, indicating that the SI-CI scheme can effectively inhibit swelling of clay present in formation.

The ultimate permeability reduction was almost same for the 3 injection schemes. This is because the clay stabilizer injected can only restrain, but not eliminate clay swelling.

The curve of permeability reduction versus cumulative volume of fluid injected can be divided into 3 stages: initial stage (0-10 PV), middle stage (10-25 PV), and final stage ( $>25$ $\mathrm{PV})$. In oilfields, the actual volume is usually not greater than $5 \mathrm{PV}$. Therefore the early stage of injection (in Fig. 15) is important for oilfield practice. At the early stage, permeability reduction in the SI-CI scheme was the lowest compared with other injection schemes. This indicates that the SI-CI scheme is effective in inhibiting clay swelling.

In the early stage of water injection, clay minerals are sensitive to fluid properties. Formation damage occurring in the early stage of injection is more severe than that occurring in the following stages of injection. It is necessary to inject high-concentration of CS to inhibit swelling of clay minerals in the early stage.

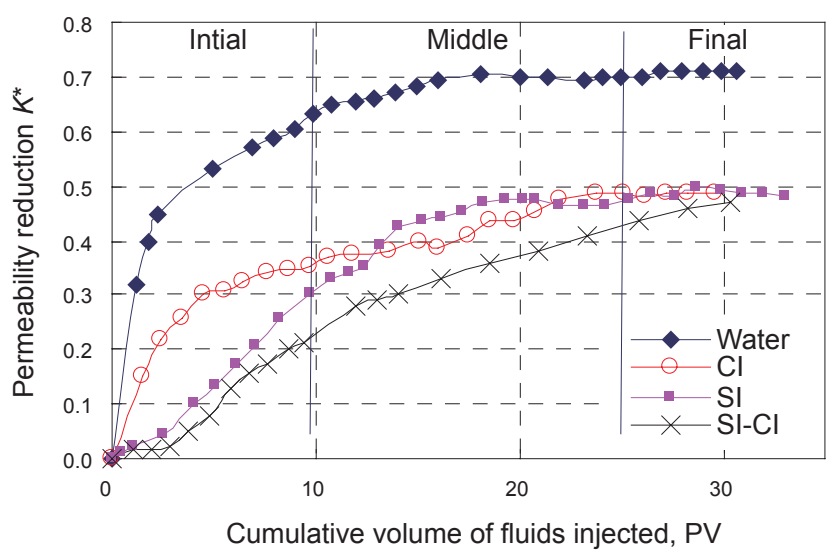

Fig. 14 Comparison between different injection schemes

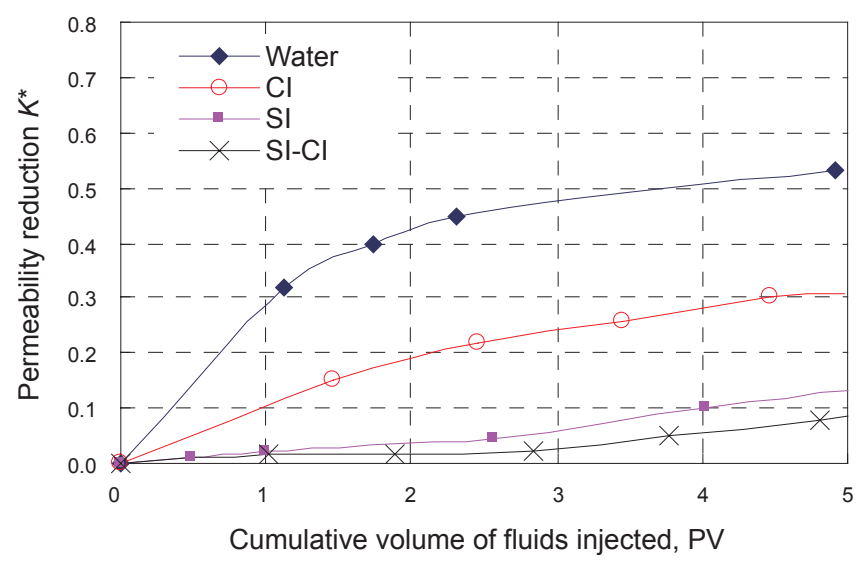

Fig. 15 Comparison of permeability reduction curves at the early stage

\section{Conclusions}

1) Performance curves for water injection wells can be used to evaluate the magnitude of formation damage. Permeability reduction $K^{*}$ and rate of wellhead pressure rise are useful parameters for evaluating formation damage.

2) In oilfields, the rate of wellhead pressure rise can also be used for treatment decision-making. For the reservoir studied in this paper, if the rate of wellhead pressure rise is greater than $0.0137 \mathrm{MPa} / 10^{3} \mathrm{~m}^{3}$ in a month, it is necessary to inject clay stabilizer.

3) There is a significant difference in permeability reduction for different injection schemes at the early stage of injection. At the end of test, the same volume of clay stabilizer was used and the ultimate values of permeability reduction were almost the same for different injection schemes of clay stabilizer.

4) To protect the formation from swelling clay damage, it is necessary to inject high concentration clay stabilizer at the beginning of water injection. Slug injection of highconcentration clay stabilizer followed by continuous injection 
of low-concentration clay stabilizer is recommended for the Bohai offshore oilfield.

\section{Acknowledgements}

The authors would like to express thanks to the Bohai offshore oilfield engineers for providing data.

\section{References}

Al-Hamadah A M. Factors affecting injectivity decline. 1995. SPE paper 29893

Bedrikovetsky P G, Fonseca D R, da Silva M J, et al. Well-history-based prediction of injectivity decline during seawater flooding. 2005. SPE paper 93885

Chang F F and Civan F. Practical model for chemically induced formation damage. Journal of Petroleum Science and Engineering. 1997. 17: 123-137

Civan F. Interpretation and correlations of clay swelling measurements. 1999. SPE paper 52134

Civan F. Water sensitivity and swelling characteristics of petroleum- bearing formations: Kinetics and correlation. 2001. SPE paper 67293 Leone J A and Scott M E. Characterization and control of formation damage during water flooding of a high-clay-content reservoir. 1988. SPE paper 16234

Moghadasi J, Muller-Steinhagen H, Jamialahma-di M, et al. Theoretical and experimental study of particle movement and deposition in porous media during water injection. Journal of Petroleum Science and Engineering. 2004. 43: 163-181

Molenaar M M and Huyghe J M. A constitutive model for swelling shales. 1996. SPE/ISRM paper 47332

Nasr-El-Din H A, A1-Mulhem A A and Lynn J D. Evaluation of clay stabilizers for a sandstone field in central Saudi Arabia. 1998. SPE paper 39584

Norman C A and Smith J E. Experience gained from 318 injection well KOH clay stabilization treatments. 2000. SPE paper 60307

Sharma M M and Pang S. Injectivity decline in water injection wells: An offshore Gulf of Mexico case study. 1997. SPE paper 38180

Xiong $\mathrm{H}$ and Holditch S A. A comprehensive approach to formation damage diagnosis and corresponding stimulation type and fluid selection. 1995. SPE paper 29513

(Edited by Sun Yanhua) 